Raquel Lázaro-Gutiérrez

Universidad de Alcalá

Emilia Iglesias-Fernández

Universidad de Granada

Gabriel Cabrera-Méndez

Universidad de Alcalá

\title{
Aspectos éticos de los protocolos de actuación en interpretación telefónica
}

Palabras clave: interpretación telefónica, protocolos de actuación, ética, habilidades, rol del intérprete

La modalidad de la interpretación telefónica está adquiriendo cada vez más importancia en los servicios públicos de España debido a las numerosas ventajas que ofrece. Fernández Pérez (2012) estableció una clasificación de las habilidades particulares que los intérpretes telefónicos deben adquirir según sus dos funciones principales: la función de coordinación del discurso y la función de traducción. Además de estas habilidades, es imprescindible que los intérpretes telefónicos conozcan en profundidad y sigan escrupulosamente los protocolos que se diseñan y desarrollan para los distintos clientes de interpretación telefónica, como aquellos que se utilizan para las líneas de emergencia. El objeto de estudio de esta contribución lo constituyen, precisamente, estos protocolos de actuación. Nuestro punto de partida son las especificaciones que deben hacerse sobre la actuación de los intérpretes, que con frecuencia divergen de o, incluso, contradicen los principios éticos más comunes, como la fidelidad o la neutralidad. Este estudio consiste en un análisis minucioso de los protocolos utilizados en la empresa Dualia Teletraducciones y en una 
reflexión crítica desde una perspectiva ética sobre los ajustes que se sugieren en ellos a propósito del rol tradicional del intérprete.

\section{Interpretación telefónica}

A pesar del reciente auge de la interpretación simultánea por videoconferencia, la interpretación telefónica ${ }^{1}$ es la variante más popular de la interpretación remota, que implica que al menos uno de los participantes en la interacción mediada no se encuentre físicamente presente junto con los demás. Algunos autores, como Braun y Taylor (2011) o Ruiz Mezcua (2018), reducen el término "interpretación remota" únicamente a aquellos casos en los que es el intérprete el que se conecta desde la distancia, mediante conexión de audio o vídeo, con los hablantes primarios de una interacción para realizar el trabajo de interpretación. Sin embargo, nosotros preferimos utilizar este término como un paraguas, englobando todos los trabajos de interpretación que implican la conexión mediante audio o vídeo de cualquiera de los participantes en la interacción, ya sean intérpretes o hablantes primarios, incluyendo las llamadas a tres (lo que Braun y Taylor (2011) denominan "interpretación por teleconferencia").

La interpretación remota se puede proporcionar en modo simultáneo, consecutivo y bilateral (Lázaro Gutiérrez, 2021), y es cada vez más frecuente gracias a su versatilidad, los avances tecnológicos cada vez más vertiginosos, y la tendencia hacia la digitalización y el teletrabajo, que ha terminado estableciéndose a causa de los cambios sociales que ha impuesto la reciente crisis sanitaria ocasionada por la pandemia de COVID-19 (Peiró y Soler, 2020).

Los servicios de interpretación telefónica se proporcionan por parte de una gran variedad de organizaciones, que pueden ser instituciones públicas, asociaciones, ONG y empresas privadas (Ruiz Mezcua, 2018). En España la situación más habitual es que organismos públicos de todo nivel y alcance (por ejemplo, ayuntamientos, servicios de salud, consejerías, etc.) y entidades privadas (grandes empresas y PYMES), contraten los servicios de las empresas de interpretación telefónica que operan en este país, entre las que se encuentran Interpret Solutions, Migralingua, Asitel, Ofilingua, Seprotec o la que participa en este estudio, Dualia Teletraducciones. Los organismos públicos contratan empresas de interpretación telefónica a través de licitaciones públicas, mientras que las empresas privadas suscriben contratos mercantiles.

1 Este artículo no trata de los cambios tecnológicos que ha experimentado la interpretación a raíz de la epidemia de COVID-19. 


\section{Condicionantes, competencias y formación en interpretación telefónica}

Para prestar servicios de interpretación telefónica, es necesario que los intérpretes desplieguen una serie de habilidades específicas. En la mayoría de los casos, los intérpretes no han recibido información previa sobre el encargo de interpretación concreto al que se van a enfrentar. Esto conlleva que carezcan de información contextual, algo que viene agravado por el hecho de no percibir información visual. Entre los elementos que componen este contexto y que se han indicado como esenciales (Lázaro Gutiérrez y Cabrera Méndez, 2019) se encuentran las personas y elementos que están presentes en la interacción, como los hablantes principales (su papel en la conversación, su lenguaje corporal, sus movimientos...), y aquellos elementos físicos a los que se refieren (por ejemplo, una camilla a la que se refieran un médico y un paciente durante una consulta).

Esta falta de contexto visual es un estresor para los intérpretes telefónicos (Andres y Falk, 2009), así como no contar con información sobre el encargo de interpretación antes de enfrentarse a él (Wilson, 2010). Aparte de esto, algunos autores como Crezee, Jülich y Hayward (2013) o Iglesias Fernández y Ouellet (2018) han achacado el estrés de los intérpretes telefónicos a sus condiciones laborales, señalando que estos intérpretes suelen trabajar como autónomos, enfrentándose a cierta irregularidad en el flujo de sus encargos y a la percepción de tarifas inferiores a las que perciben los intérpretes presenciales de conferencias. El uso de la tecnología supone una dificultad añadida (Pertusa Elorriaga, 2012) y distinta a la que puede provocar el uso de la tecnología de interpretación simultánea en cabina. Los intérpretes telefónicos suelen contar con buenos equipos (teléfono, ordenador, auriculares, micrófono, etc.) y procuran realizar los servicios de interpretación en un entorno tranquilo y adecuado. Sin embargo, sufren en soledad aquellos problemas técnicos que se producen por su parte y también aquellos que les surgen a los hablantes primarios, que pueden estar utilizando equipos viejos o inadecuados, o pueden encontrarse en lugares donde no hay suficiente cobertura o son ruidosos. Lázaro Gutiérrez y Cabrera Méndez (2019) también señalan el uso inadecuado que los hablantes primarios, debido a su falta de costumbre, hacen del equipo, por ejemplo, al utilizar la función de manos libres de un teléfono móvil que dejan en una mesa mientras varios hablantes interactúan entre sí en una habitación. El micrófono del dispositivo no es capaz de captar con nitidez todas las voces, particularmente si no se dirigen al aparato. Por otro lado, se ha observado en llamadas a tres que el sonido que llega a los intérpretes desde los usuarios finales es, en ocasiones, más bajo y menos nítido 
que el que llega a los operadores o proveedores de servicios, con el consiguiente impacto en la calidad del servicio.

El ritual de establecimiento de comunicación y la coordinación del discurso son también aspectos característicos de esta modalidad de interpretación. Cuando un usuario quiere utilizar los servicios de un intérprete telefónico, tiene que seguir unos pasos específicos consistentes, como mínimo, en llamar a un número de teléfono determinado y seguir las instrucciones de un contestador automático para seleccionar una serie de opciones hasta llegar a hablar con un intérprete. Mientras todo esto ocurre, es posible que el otro hablante primario se encuentre junto con este usuario sin entender nada de lo que está pasando. Una vez que el intérprete entra en escena, se hace imprescindible que explique a ambas partes cuál es su papel y cómo se va a desarrollar la interacción. Durante la interacción será responsable de aclarar a ambas partes turnos demasiado largos, interrupciones, solapamientos o conversaciones laterales de diversa índole, desde peticiones de información adicional y explicaciones hasta interpelaciones y negociaciones (Lázaro Gutiérrez y Cabrera Méndez, 2017). El intérprete se somete a la gestión de turnos del interlocutor con mayor rango dentro de la conversación, en cuya cúspide suelen situarse médicos o agentes de policía.

La formación específica en interpretación telefónica es creciente, si bien sigue proporcionándose, en su mayor parte, a través de las empresas de interpretación telefónica, tanto como formación inicial como continua (Lázaro Gutiérrez, 2021). Siendo una modalidad reciente que requiere el uso de tecnología, muchas universidades no se encuentran preparadas en cuanto a infraestructuras o personal para proporcionar formación en esta área, por lo que se suele impartir como cursos complementarios.

En los últimos años, se han elaborado varias propuestas de formación en interpretación telefónica. Destaca la iniciativa de Fernández Pérez (2012, 2015), que, previamente a proponer un conjunto de actividades basadas en juegos de rol, realizó una investigación exhaustiva para definir las características de la interpretación telefónica y clasificar las habilidades específicas, que atribuyó a dos roles principales: papel de coordinador, en cuanto a que organiza el principio y el final del encuentro y los turnos de palabra, y el papel de traductor. Transversales para ambos roles son las habilidades para manejar elementos prosódicos y el uso de los estilos directo e indirecto.

Entre los aspectos característicos de la interpretación telefónica, Lázaro Gutiérrez (2021) señala el uso de protocolos adaptados a diferentes clientes. Estos 
protocolos sirven para afrontar aquellos condicionantes más difíciles de esta modalidad de interpretación (como la falta de información sobre el cliente, procedimiento ante posibles eventos inesperados, tratamiento de las partes participantes...) y para atajar sus posibles consecuencias. Además, los protocolos son la base para la formación continua de los intérpretes. Una vez elaborados por parte de las empresas de interpretación telefónica a partir de las características del contrato que se firma con los clientes finales, se distribuyen entre los intérpretes a la vez que se les proporcionan otras directrices y se les ofrece formación en línea por videoconferencia.

\section{3 Ética e interpretación telefónica}

Los intérpretes profesionales se rigen por códigos deontológicos, que contienen una lista de principios éticos generales (Valero Garcés, 2017) que se aplican a la profesión. Siguiendo a Baixauli (2012), el objetivo principal de los códigos deontológicos es fomentar la autonomía moral. Además, estos documentos desempeñan multitud de propósitos, por ejemplo, sirven como guía de lo que se puede o debe hacer, ya que los intérpretes profesionales se comprometen a seguirlos, o como documento vinculante en el caso de sanción por incumplimiento. Los códigos deontológicos pueden considerarse instrumentos didácticos para formar intérpretes; como criterio de contratación; como instrumento de control de calidad; como elemento de cohesión interna; o como instrumento legitimador social. Como indica Brander de la Iglesia (2017), la deontología, por su parte, se encuentra íntimamente ligada a otros dos conceptos fundamentales: la ética (general), que es la rama de la filosofía que se ocupa de la moral (Etxeberría, 2002) junto con la ética aplicada, que lidia con los problemas morales de la sociedad y de las profesiones, y la metaética, que constituye la manera en que pensamos y hablamos de la ética. Camayd-Freixas (2013) indica que la ética no debería abordarse únicamente desde la deontología y que resultaría conveniente aplicar a la práctica de la interpretación otras teorías complementarias, como el consecuencialismo (en concreto, el utilitarismo de Benthan, por el que un acto es moralmente aceptable si sus previsibles consecuencias aportan el mayor de los beneficios, la mayor felicidad, al mayor número posible de personas), los sentimientos morales (empatía, compasión, benevolencia y afecto) o la ética de la virtud (seguir valores positivos previamente definidos).

Por otro lado, Ibarra Rosales (2007: 44) señala que "la ética profesional es una ética de valores", en cuanto a que los principios de esta ética tienen como 
referente los valores profesionales por los que opta libremente el profesional al asumir un ejercicio profesional ético. No obstante, es necesario tener en cuenta que podemos aproximarnos a los principios éticos de distintas maneras. Podemos, por ejemplo, partir de la enunciación de los principios éticos para guiar las actuaciones de los intérpretes (siguiendo un enfoque normativo), o, por el contrario, podemos presentar casos de dificultades o problemas éticos para reflexionar sobre la actuación moralmente ideal ante ellos (con un enfoque heurístico). En la actualidad se prefiere un modelo mixto (Brander de la Iglesia, 2017; Páez Gallego, 2015), pero es inevitable que, aunque se utilicen tanto principios teóricos como casos prácticos para definir actuaciones éticamente correctas, se parta a veces desde unos y a veces, desde otros. Mientras que las asociaciones de intérpretes y las instituciones formativas suelen partir de principios teóricos, como veremos en esta contribución, a la hora de elaborar protocolos de actuación, las empresas de interpretación telefónica parten de situaciones comunicativas habituales (descritas, bien mediante la observación, bien mediante entrevistas con los clientes) para definir actuaciones y proporcionar instrucciones a propósito de dificultades éticas anticipadas.

Considerar el código deontológico de una profesión como indiscutible puede resultar práctico para estudiantes de interpretación cuando todavía están en formación. También se da el caso de que los profesores presentan ante los estudiantes contenidos excesivamente teóricos, por lo que ocurre que, como indican Baker y Maier (2011), se enseña a los estudiantes a seguir los principios éticos de manera incuestionable, fallando, muchas veces, a la hora de ayudar a desarrollar un entendimiento profundo de los aspectos éticos. Aún hoy se oyen testimonios de estudiantes sobre la distancia entre los contenidos académicos de la universidad y la realidad profesional. Esto es particularmente pertinente en ámbitos de la interpretación en los que confluyen distintos enfoques éticos, como en la interpretación en los servicios públicos, en la que los intérpretes se rigen por los principios éticos de la interpretación a la vez que por los del entorno en el que interpretan, por ejemplo, en el ámbito médico (Lázaro Gutiérrez, 2020) o en del funcionariado (Valero Garcés, 2017), por ejemplo.

La existencia de un código deontológico para una actividad concreta es indicativa del grado de profesionalización de esta actividad (Phelan, 2020; Skaaden, 2020). Sin embargo, en el caso de la interpretación en los servicios públicos, podemos observar que, dado su bajo grado de profesionalización, existen carencias en la oferta formativa o en el establecimiento de códigos deontológicos específicos. Phelan (2020) señala que, en este caso, los códigos deontológicos 
suelen emanar de asociaciones profesionales de diferentes grados de especialización. Los códigos de asociaciones coexisten con códigos de empresas privadas en aquellos países como España, Irlanda y el Reino Unido, donde la interpretación en los servicios públicos se encuentra externalizada. Paralelamente, varias normas ISO incluyen aspectos éticos (ISO 13611:2014 Interpreting Guidelines for community interpreting, ISO 20228:2019 Interpreting services - Legal interpreting - Requirements, ISO 21998:2020 Interpreting services - Healthcare interpreting - Requirements and recommendations).

En esta contribución, queremos abundar en la deontología aplicada a la interpretación telefónica, un campo todavía no suficientemente explorado de manera independiente a la interpretación presencial, para revelar los ajustes que se producen en esta modalidad de interpretación respecto a los principios éticos tradicionales, como fidelidad, confidencialidad, neutralidad o profesionalidad. Centrándose también en la interpretación remota, en concreto en la proporcionada a través de videoconferencia en contextos legales, Devaux (2017) señala que, si bien muchas dificultades éticas aparecen por igual en tanto en la interpretación presencial como en la remota, otras son características de o se ven exacerbadas en esta última.

En general, sobre aspectos éticos en interpretación telefónica, se ha dicho que el hecho de que los intérpretes no se encuentren físicamente presentes en la interacción contribuye a mantener la confidencialidad y la privacidad de los usuarios finales (Hewitt, 1995; Kelly, 2008; Wadensjö, 1999; Rosenberg, 2004; Phillips, 2013; Ko, 2006; Mikkelson, 2003). En el ámbito sanitario, donde la interpretación telefónica es especialmente popular, gracias a esta modalidad se evitan situaciones que sí se dan en la presencial, cuando los intérpretes tengan que mantenerse al otro lado de una puerta entreabierta o una cortina, o tengan que darse la vuelta cuando se realiza la anamnesis (Lázaro Gutiérrez, 2021). Del mismo modo, es menos probable en interpretación telefónica que el intérprete y el usuario final se queden a solas sin la presencia del proveedor de servicios, lo que evita ocasiones en que se puedan producir conversaciones personales entre ellos (Lázaro Gutiérrez, 2018, 2021).

Por otro lado, como menciona Devaux (2020) la modalidad remota introduce ciertas peculiaridades que afectan a determinados aspectos éticos. La clásica presentación del intérprete, donde se da la oportunidad para explicar aspectos éticos como la confidencialidad o la imparcialidad y se establecen límites de actuación, a veces no se produce. Además, Devaux (2020) observó que los intérpretes remotos se apartan con más frecuencia del papel de mero intérprete 
para proporcionar aclaraciones o alertar de problemas en el flujo de la comunicación, en ocasiones, problemas tecnológicos.

Este artículo pretende contribuir a ahondar en aquellos aspectos éticos que, como señalaba Devaux (2020) son peculiares en la interpretación telefónica. Participa en el estudio la empresa Dualia Teletraducciones, que presta servicio $24 \mathrm{~h}$ de interpretación telefónica desde 2004 en España, tanto en los servicios públicos como para empresas privadas. La empresa se encuentra actualmente suscrita al código deontológico de ANETI (Asociación Nacional de Empresas de Traducción e Interpretación) y redacta protocolos de actuación que requieren la ratificación de los clientes antes de su distribución al equipo de intérpretes a través de su aula virtual.

\section{Metodología y objeto de estudio}

Además de las habilidades que se describen al principio de este artículo, es imprescindible que los intérpretes telefónicos conozcan en profundidad y sigan escrupulosamente los protocolos de actuación que se diseñan y desarrollan para los distintos clientes de interpretación telefónica, como aquellos que se utilizan para las líneas de emergencia, centros de salud, servicio a los mayores o de violencia de género. El objeto de estudio de esta contribución lo constituyen, precisamente, estos protocolos de actuación. Nuestro punto de partida son las especificaciones que deben hacerse sobre la actuación de los intérpretes, que con frecuencia divergen de o, incluso, contradicen los principios éticos más comunes, como la fidelidad o la neutralidad. Esto es así porque los códigos deontológicos utilizados para la interpretación suelen ser superficiales y no están adaptados a ámbitos o clientes concretos (Devaux, 2017). Además, como indica Kermit (2020), si tomamos en consideración aquellos principios éticos sobre los que suele haber consenso entre los intérpretes profesionales, podemos descubrir que existe cierta distancia entre lo que es ético y lo que es técnicamente posible.

Este estudio consiste en un análisis minucioso de los protocolos utilizados en la empresa Dualia Teletraducciones y en una reflexión crítica desde una perspectiva ética sobre los ajustes que se sugieren en ellos a propósito del rol tradicional del intérprete. El análisis se articula en torno a los principios éticos clásicos de neutralidad, confidencialidad, fidelidad y profesionalidad, que se describen para posteriormente contrastar con ellos los protocolos de actuación de la empresa Dualia Teletraducciones. 
Los protocolos de actuación de la empresa Dualia Teletraducciones son documentos breves que de manera sucinta proporcionan guía sobre la actuación particular en el servicio a un cliente concreto. Consisten de una o dos páginas con instrucciones breves de una sola frase, en español y adaptadas a la comprensión de todos los intérpretes de todas las lenguas, independientemente de su nivel de competencia en la lengua española. Estos documentos no se utilizan de manera aislada, sino que se complementan con el "kit de bienvenida", el código deontológico de la empresa, las píldoras formativas (pequeños documentos o vídeos que se centran en dificultades concretas que previamente han sido identificadas en el seno del Departamento de Calidad) y los foros de los intérpretes, en los que, con frecuencia, surgen hilos relacionados con aspectos éticos. Todos estos elementos se hacen llegar a los intérpretes a través del aula virtual de Dualia Teletraducciones.

Estos protocolos, como no puede ser de otro modo, son estacionales y actualizables, ya que entran en vigor con cada contrato que la empresa suscribe con sus clientes. Se elaboran a partir de los contratos suscritos, así como de entrevistas y reuniones mantenidas con los responsables de los servicios. Una vez recibidos, los intérpretes son responsables de leerlos y deben firmarlos comprometiéndose a seguirlos. En este artículo se va a analizar un total de 5 protocolos, actualmente en vigor, para los siguientes clientes: el servicio de teleasistencia para personas mayores de la Junta de Andalucía ( $\left.\mathrm{P}_{1}\right)$, el teléfono nacional de atención a víctimas de violencia de género $016\left(\mathrm{P}_{2}\right)$, el teléfono de emergencias 112 de Andalucía ( $\left.\mathrm{P}_{3}\right)$, Cruz Roja Española $\left(\mathrm{P}_{4}\right)$ y el Centro COMETA ( $\mathrm{P}_{5}$ ), encargado de la supervisión telefónica de agresores portadores de dispositivos de localización (pulseras en el tobillo).

Los documentos consisten en una descripción del servicio de los clientes, de la naturaleza y estructura de las llamadas más frecuentes, las características del lenguaje que se utiliza, y sobre el tipo de interpretación que se debe realizar. Así, en el $\mathrm{P}_{5}$ encontramos una descripción detallada sobre el Centro COMETA y sus funciones y aprendemos en qué consiste el sistema de supervisión telefónica de agresores, incluyendo la pena de prohibición de aproximación (quién la impone y en qué consiste), cómo funciona la tecnología en que se basa el sistema (distancia de alejamiento), las características del dispositivo (cómo funciona, se instala, configura y mantiene) y qué tipo de avisos se generan.

En cuanto a la naturaleza y estructura de las conversaciones, en el $\mathrm{P} 1$ podemos ver la casuística de las llamadas del servicio de teleasistencia a personas mayores, que incluyen propósitos tan diversos como recordatorios de medicación, 
saludar si hace tiempo que no se ha contactado con el usuario, llamadas a urgencias médicas, o para solicitar una cita con un médico, averías con el dispositivo, felicitaciones de cumpleaños y del santo, preguntas por la evolución tras una cirugía reciente o llamadas a los vecinos o familiares que tienen llave de la casa del usuario. En este mismo protocolo, se indica a los intérpretes que es fundamental actuar con paciencia de cara a las dificultades que los usuarios finales del servicio tienen con el lenguaje, debido a problemas de audición o deterioro cognitivo. Se recomienda una pronunciación y articulación extremadamente cuidadas, utilización de oraciones sencillas y precisas, y confirmar la comprensión del usuario final tras cada intervención.

\section{$5 \quad$ Análisis}

Algunas de las recomendaciones y directrices que figuran en los protocolos se relacionan con principios éticos clásicos de los códigos deontológicos de los intérpretes, como son la confidencialidad, la neutralidad, la fidelidad y la profesionalidad. En este estudio analizamos cómo se abordan estos cuatro principios en los protocolos de actuación de la empresa Dualia Teletraducciones.

\subsection{Neutralidad}

La neutralidad es una piedra angular de los códigos deontológicos para la interpretación. Habitualmente se contrapone la neutralidad a la abogacía y las tareas de mediación que tan comunes son en algunos de los ámbitos de la interpretación en los servicios públicos, como en el sanitario (Angelelli, 2004). Incluso en ámbitos tan restringidos como el judicial, hay intérpretes profesionales que indican que, en ocasiones, tienen que realizar labores de mediación cuando, por ejemplo, los interpretados acuden nerviosos a un juicio y el intérprete les tranquiliza dentro de su rol de coordinador (Wadensjö, 1998) para poder establecer la comunicación (Valero Garcés, 2017).

En los protocolos que analizamos en este artículo podemos encontrar directrices que contravienen el principio de neutralidad y promueven un rol para los intérpretes más cercano a la mediación. Por ejemplo, en el $\mathrm{P}_{1}$, se pide a los intérpretes que muestren "un extra de empatía" hacia los mayores, usuarios finales del servicio, y en el $\mathrm{P}_{2}$ se solicita que se trate a las víctimas de violencia de género con respeto y también con cariño. Además, tanto en este protocolo como en el $\mathrm{P}_{4}$ se reconoce la posibilidad de que los usuarios finales, al estar pasando por momentos personales muy difíciles, se desahoguen con los 
intérpretes cuando empiezan a hablar con ellos, ya que, en muchas ocasiones, se trata de la primera vez que pueden hablar con un compatriota o, por lo menos, con alguien que habla su misma lengua, y sienten la necesidad de contar su historia proporcionando información y detalles que van mucho más allá de la respuesta a la pregunta que originalmente había planteado el proveedor de servicios. En estos casos, tras haberlo pactado con los proveedores de servicios, se propone dejar a los usuarios finales hablar libre y tranquilamente y los intérpretes, por su parte, no les interrumpirán, sino que, una vez hayan acabado, proporcionarán al proveedor un resumen lo más concreto posible.

El "kit de bienvenida" del intérprete de Dualia Teletraducciones, que incluye directrices básicas sobre la actuación de los intérpretes para encargos de esta empresa, señala que, de preferencia, debe utilizarse la tercera persona en vez de la primera. Como sugiere Tryuk (2017), el uso de la primera persona es una marca tanto de neutralidad como de fidelidad. Al transmitir un mensaje en primera persona, su forma se acerca más a la de los enunciados originales. Del mismo modo, utilizar la primera persona al trasladar los mensajes de ambos interlocutores implica evitar referencias a unos y a otros, reforzando la distancia y la sensación de que el intérprete es un mero conducto sin voz propia. Utilizar la tercera persona hace más visible al intérprete, lo que puede dar lugar a fomentar conversaciones paralelas con ambos hablantes primarios, ya que estos tienden a dirigirse al intérprete en vez de directamente a su interlocutor. Por otro lado, se asemeja más al comportamiento natural de una conversación telefónica y solventa el quebrantamiento sistemático de la cuarta pared de la interpretación con aclaraciones del intérprete cuando hay problemas de comunicación en alguna de las líneas participantes en la llamada.

\subsection{Confidencialidad}

La confidencialidad es un principio básico de muchos códigos deontológicos. Si bien parece haber consenso respecto al mantenimiento de la confidencialidad en cualquier ámbito de la interpretación, Valero Garcés (2017) señala como ejemplo el caso de un intérprete al que, durante una conversación paralela, le fue confesado un delito por parte de la persona para la que interpretaba. En estos casos, los intérpretes profesionales señalan como deber personal el poner en conocimiento de las autoridades policiales o judiciales dicho hecho. Es dudoso que la confidencialidad se esté rompiendo en estos casos, ya que los intérpretes tienen también la obligación de interpretar todo lo que se diga de acuerdo con el principio de fidelidad, pero los principios éticos a veces se solapan y cabe 
la posibilidad de que se produzcan dificultades, o incluso, dilemas éticos, que pongan a prueba la profesionalidad e integridad de los intérpretes.

En el caso que nos ocupa, no existe ningún aspecto en los protocolos analizados que sugiera cualquier vulneración del principio de confidencialidad. Muy al contrario, se enfatiza y se recuerda a los intérpretes que la confidencialidad debe siempre mantenerse y, bajo ningún concepto, se debe preguntar por datos personales a no ser que el proveedor de servicios así lo requiera.

\subsection{Fidelidad}

El principio de fidelidad, junto con el de neutralidad, se ve afectado cuando el intérprete desempeña tareas más cercanas a la mediación y ha sido el objeto de numerosos estudios en el ámbito de la interpretación en los servicios públicos (ver, por ejemplo, Valero Garcés, 2007 en el ámbito penitenciario o Angelelli, 2004 en el ámbito sanitario). En este caso, son numerosas las sugerencias que figuran en los protocolos que proponen que los intérpretes intervengan con voz propia para añadir información, contribuir activamente a que no se produzcan malentendidos o resumir las intervenciones de los usuarios finales. Por ejemplo, en el $\mathrm{P}_{1}$ se indica que los intérpretes deben requerir confirmación de que los usuarios finales han entendido el mensaje en cada intervención. Se reconoce la posibilidad de que se tengan que establecer diálogos entre los intérpretes y los usuarios finales causados por algún problema de comprensión. En este caso, los intérpretes solucionan, en la medida de lo posible, el problema de comprensión con los usuarios finales y trasladan un resumen tanto del percance como de la solución al proveedor de servicios.

Meyer (2012) averiguó que, en conversaciones médicas mediadas por intérpretes, el personal sanitario tiende a acomodar menos el lenguaje al nivel de comprensión de sus pacientes, recayendo esta responsabilidad en el intérprete. El P1 se hace eco de esta circunstancia y sugiere a los intérpretes trasladar los mensajes con frases sencillas y cortas, con estructuras precisas y adecuando el mensaje a las convenciones culturales de los usuarios finales. Del mismo modo, los intérpretes deben repetir o reformular mensajes si perciben que los usuarios finales no los han comprendido correctamente.

En el P2, además de trasladar todo lo que se diga de manera fiel, se solicita que los intérpretes también informen si perciben algunos detalles como un cambio en la actitud de la víctima, que su discurso no sea coherente, o si se detectan más personas en la conversación. En casos de llamadas de víctimas 
de violencia de género al teléfono de emergencia es una cuestión de seguridad conocer estos detalles, ya que pueden ser indicadores de que el agresor se encuentre presente y la víctima en peligro. Además, cabe la posibilidad de que, en este número, al ser gratuito, se reciban llamadas por parte de bromistas. Se solicita a los intérpretes que, si perciben algo que indique que estas alertas son falsas, comuniquen sus sospechas a los proveedores del servicio.

En el $\mathrm{P}_{4}$ se hace referencia explícita a las barreras culturales, que no se resuelven simplemente con un traslado lingüístico. En estos casos, los intérpretes tienen permiso para intervenir con voz propia y explicarlo al personal de Cruz Roja.

En el caso de las llamadas al $112\left(\mathrm{P}_{3}\right)$ se prioriza la rapidez y la agilidad. Por este motivo, los intérpretes deben trasladar el mensaje de manera concisa, resumiendo si es necesario e, incluso, interrumpiendo al usuario final para transmitir detalles cruciales lo antes posible. Los resúmenes, por el contrario, están totalmente contraindicados en el $\mathrm{P}_{5}$, particularmente cuando se traslada lenguaje malsonante e insultos. Si bien en el $\mathrm{P}_{3}$ no se traslada el contenido exacto de los insultos, sino que, simplemente, se indica al proveedor que su interlocutor está insultando o se expresa con lenguaje malsonante, en el $\mathrm{P}_{5}$ el contenido de los insultos es crucial, ya que pueden constituir amenazas de agresores contra víctimas y es fundamental que los proveedores del servicio cuenten con esta información para actuar en consecuencia.

\subsection{Profesionalidad}

Aunque la profesionalidad se incluye como principio en multitud de códigos éticos para intérpretes, como puede ser el de la Asociación de Interpretes de Conferencia de España, autores como Bancroft (2017) reconocen que existe variedad respecto a lo que se entiende como requisitos de profesionalidad. En general, la profesionalidad engloba comportamientos que fomentan mantener la calidad y la dignidad de la profesión. Este principio, por lo tanto, desaconseja aceptar encargos que no se puedan realizar con calidad o comportamientos de diversa índole que perjudiquen a intérpretes (su imagen o sus condiciones laborales) o clientes (su integridad personal en un sentido amplio o la calidad del servicio que reciben).

El comportamiento profesional del intérprete se refuerza y enfatiza de manera general en todos los protocolos. Se recuerda a los intérpretes que no se deben atender servicios entrantes si no se reúnen las condiciones de ausencia de ruido y terceras personas, disponibilidad de tiempo y disponibilidad técnica 
(suficiente batería en el teléfono u ordenador, por ejemplo). Del mismo modo, se pone énfasis en que los intérpretes, a pesar de que en algunas ocasiones tengan que interpretar llamadas que constituyen alertas falsas o bromas, o en las que el comportamiento de los usuarios finales es irrespetuoso, deben actuar con paciencia, corrección y amabilidad.

Por otro lado, en el $\mathrm{P}_{1}$ se solicita a los intérpretes que realicen una presentación muy resumida. Esto se debe a las características de los usuarios finales del servicio, para los que hay que expresarse con mucha sencillez y concisión. Por otro lado, en el $\mathrm{P}_{3}$ se aconsejan las interrupciones cuando los alertantes se extienden en una explicación llena de detalles para trasladar cuanto antes el mensaje a los proveedores del servicio.

\section{Conclusiones}

Entre los aspectos característicos de la interpretación telefónica se encuentra el uso de protocolos adaptados a diferentes clientes (Lázaro Gutiérrez, 2021). En este artículo nos hemos ocupado de la deontología de la interpretación telefónica, en concreto, de los ajustes que es necesario hacer en los protocolos respecto a los principios éticos tradicionales de la interpretación, como la fidelidad, la confidencialidad, la neutralidad o la profesionalidad. Nuestro punto de partida han sido las especificaciones que figuran en los protocolos de actuación de la empresa Dualia Teletraducciones respecto a la actuación de los intérpretes, que con frecuencia divergen de o, incluso, contradicen estos principios, denotando la necesidad de una actualización y la reconsideración de la forma en la que esta profesión se enseña en las universidades. Las mayores diferencias encontradas están relacionadas con la gestión de turnos de palabra (tarea que los códigos deontológicos asignan a los interpretes pero que en la práctica real recae sobre los profesionales que hacen uso del servicio de interpretación) y el concepto de la defensa del usuario alófono, hecho que se diluye en estos protocolos, que, quizás, concede nás importancia a considerar el entendimiento del profesional que reclama el servicio sobre la comprensión del usuario extranjero. Estas divergencias, si bien a veces son más frecuentes en la interpretación telefónica, como la preferencia por usar la tercera persona, no son exclusivas de esta modalidad, encontrándose presentes también en la interpretación presencial en los servicios públicos (valgan como ejemplo las intervenciones del intérprete con voz propia para explicar aspectos culturales). En los protocolos analizados, además, se refuerzan actuaciones que se acercan a la mediación intercultural, como la adaptación del discurso al perfil de los usuarios, la intervención de los intérpretes para dar explicaciones tanto a los proveedores 
de servicios como a los usuarios finales, la producción propia de repeticiones y reformulaciones o las muestras de empatía.

\section{Bibliografía}

Andres, D., Falk., S. (2009): «Information and communication technologies (ICT) in interpreting-Remote and telephone interpreting». En: Andres Dörte, Sonja Pöllabauer (eds.), Spürst Du wie der Bauch rauf runter? Fachdolmetschen im Gesundheitsbereich /Is everytbing all topsy turvy in your tummy? Health care interpreting. Munich: Martin Meidenbauer, 9-27.

Angelelli, C. (2004): Revisiting the Interpreter's Role: A Study of Conference, Court, and Medical Interpreters in Canada, Mexico and the United States. Amsterdam: John Benjamins.

Baixauli Olmos, L. (2012): La interpretació als serveis públics desde una perspectiva ética. La deontología professional i l'aplicació al context penitenciari. Castellón de la Plana: Universidad Jaume I.

Baker, M., Maier, C. (2011): «Ethics in interpreter and translator training». The Interpreter and Translator Trainer, 5, 1, 1-14.

Bancroft, M. (2017): «The Voice of Compassion: Exploring Trauma-Informed Interpreting». En: Carmen Valero-Garcés, Rebecca Tipton (eds.), Ideology, Etbics and Policy Development in Public Service Interpreting and Translation. Bristol: Multilingual Matters, 195-219.

Brander de la Iglesia, M. (2017): «'A Sea of Troubles': Ethical Dilemmas from War Zones to the Classroom». En: Carmen Valero-Garcés, Rebecca Tipton (eds.), Ideology, Etbics and Policy Development in Public Service Interpreting and Translation. Bristol: Multilingual Matters, 85-101.

Braun, S., Taylor, J. L. (2011): «Video-mediated interpreting: an overview of current practice and research». En: Sabine Braun, Judith L. Taylor (eds.), Videoconference and remote interpreting in criminal proceedings. Guildford: University of Surrey, 27-57.

Camayd-Freixas, E. (2013): «Court interpreter ethics and the role of professional organization». En: Christina Schäffner, Krzysztof Kredens, Ivonne Fowler (eds.), Interpreting in a Changing Landscape. Amsterdam y Filadelfia: John Benjamins, 15-30.

Crezee, I., Jülich, S., Hayward, M. (2013): «Issues for interpreters and professionals working in refugee settings». Journal of Applied Linguistics and Professional Practice, 8 (3), 253-273. 
Devaux, J. (2017): «Virtual Presence, Ethics and Videoconference Interpreting: Insights from Court Settings». En: Carmen Valero-Garcés, Rebecca Tipton (eds.), Ideology, Etbics and Policy Development in Public Service Interpreting and Translation. Bristol: Multilingual Matters, 131-150.

Etxeberría, X. (2002): Temas Básicos sobre ética de las Profesiones. Bilbao: Desclée de Brower.

Fernández Pérez, M. M. (2012): Identificación de las destrezas de la interpretación telefónica. La Laguna: Universidad de La Laguna.

Fernández Pérez, M. M. (2015): «Propuestas de ejercicios de simulación para la didáctica de la interpretación telefónica». MonTI Special Issue, 2, 259-279.

Hewitt, W. E. (1995): Court interpretation: Model guides for policy and practice in the state courts. Williamsburg, Virginia: National Center for State Courts.

Hortal Alonso, A. (2002): Ética general de las profesiones. Bilbao: Desclée de Brouwer.

Ibarra Rosales, G. (2007): «Ética y valores profesionales». Reencuentro, 49, 43-50.

Iglesias Fernández, E., Ouellet, M. (2018): «From the phone to the classroom: categories of problems for telephone interpreting training». The Interpreters' Newsletter, 23, 19-44.

Kelly, N. (2008): Telephone interpreting: A comprehensive guide to the profession. Canada: Trafford.

Kermit, P. S. (2020): «Introduction». En: Mary Phelan, Mette Rudvin, Hanne Skaaden, Patrick Stefan Kermit (eds.), Ethics in Public Service Interpreting. Londres y Nueva York: Routledge.

Ko, L. (2006): «The need for long-term empirical studies in remote interpreting research: A case study of telephone interpreting». Linguistica Antverpiensia, 5, 325-338.

Lázaro Gutiérrez, R. (2018) «Interpretación sanitaria para víctimas de violencia de género».Panace@, XIX, 47, 96-105.

Lázaro Gutiérrez, R. (2020): «Fidelidad, confidencialidad y empatía en consultas médicas con víctimas de violencia de género mediadas por un intérprete». En: Encarnación Postigo Pinazo (ed.), Interpreting in a Changing World: New Scenarios, Technologies, Training Challenges and Vulnerable Groups. Berlín: Peter Lang. 49-64. 
Lázaro Gutiérrez, R. (2021): «Remote (telephone) interpreting in healthcare settings». En: Şebnem Susam-Saraeva, Eva Spišiaková (eds.), The Routledge Handbook of Translation and Health. Londres y Nueva York: Routledge, 216-231.

Lázaro Gutiérrez, R., Cabrera Méndez, G. (2019): «Context and pragmatic meaning in telephone interpreting». En: Pilar Garcés-Conejos Blitvich, Lucía Fernández-Amaya, María de la O Hernández-López (eds.), Tecbnology Mediated Service Encounters. Amsterdam: John Benjamins. 45-68.

Meyer, B. (2012): «Ad hoc interpreting for partially language-proficient patients: Participation in multilingual constellations». En: Claudio Baraldi, Laura Gavioli (eds.), Coordinating Participation in Dialogue Interpreting. Amsterdam y Filadelfia: John Benjamins, 99-113.

Mikkelson, H. (2003): «Telephone interpreting: Boon or Bane?» En: Luis Pérez González (ed.), Speaking in tongues: Language across contexts and users. Valencia: Universitat de València, 251-269.

Páez Gallego (2015): «Teorías normativas y descriptivas de la toma de decisiones: un modelo integrador». Opción, 31, 2, 854-865.

Peiró, J. M., Soler, A. (2020): El impulso al teletrabajo durante el COVID-19 y los retos que plantea. Geralitat Valenciana: IVIE.

Pertusa Elorriaga, I. (2012): «La interpretación en la distancia». Suplemento: la traducción y la interpretación en los servicios públicos. Revista Traditori, 03 , 25-29.

Phelan, M. (2020): «Codes of ethics». En: Mary Phelan, Mette Rudvin, Hanne Skaaden, Patrick Stefan Kermit (eds.), Etbics in Public Service Interpreting. Londres y Nueva York: Routledge.

Phillips, C. (2013): «Remote telephone interpretation in medical consultations with refugees: Meta-communication about care, survival and selfhood». Journal of Refugee Studies, 26, 4, 505-523.

Rosenberg, B. A. (2004): «A data driven analysis of telephone interpreting». En: Cecilia Wadensjö, Birgitta Englund Dimitrova, Anna-Lena Nilsson (eds.), The critical link 4: Professionalisation of interpreting in the community. Amsterdam: John Benjamins, 65-77.

Ruiz Mezcua, A. (2018): «General Overview of Telephone Interpretation (TI): A State of the Art». En: Aurora Ruiz Mezcua (ed.), Approaches to Telephone Interpretation. Research, Innovation, Teaching and Transference. Bern: Peter Lang, 7-17. 
Skaaden, H. (2020): «Ethics and profession». En: Mary Phelan, Mette Rudvin, Hanne Skaaden, Patrick Stefan Kermit (eds.), Etbics in Public Service Interpreting. Londres y Nueva York: Routledge.

Tryuk, M. (2017): «Conflict. Tension. Agression. Ethical Issues in Interpreted Asylum Hearings at the Office for Foreigners in Warsaw». En: Carmen Valero-Garcés, Rebecca Tipton (eds.), Ideology, Etbics and Policy Development in Public Service Interpreting and Translation. Bristol: Multilingual Matters, 179-194.

Valero Garcés, C. (2017): «Ethical Codes and Their Impact on Prison Communication». En: Carmen Valero-Garcés, Rebecca Tipton, R. (eds.), Ideology, Etbics and Policy Development in Public Service Interpreting and Translation. Bristol: Multilingual Matters, 105-130.

Wadensjö, C. (1998): Interpreting as interaction. London and New York: Addison Wesley Longman.

Wadensjö, C. (1999): Telephone interpreting and the synchronization of talk in social interaction». The Translator, 5, 2, 247-264.

Wilson, C. W. (2010): Working through, with or despite technology? A study of interpreter-mediated encounters when interpreting is provided by video conferencing link. Paper presented at the Critical Link 6: Interpreting in a Changing Landscape, Birmingham, UK.

\section{Ethical aspects of telephone interpreting protocols}

Keywords: telephone interpreting, protocols, ethics, skills, interpreter's role

The modality of telephone interpreting is becoming increasingly important in Spain's public services due to the numerous advantages it offers. Fernández Pérez (2012) established a classification of the particular skills that telephone interpreters must acquire based on their two main functions: the speech coordination function and the translation function. In addition to these skills, it is essential that telephone interpreters have a thorough knowledge of and scrupulously follow the protocols that are designed and developed for the different telephone interpreting clients, such as those used for emergency lines.

The object of study of this contribution is precisely these protocols. Our starting point is the specifications to be made regarding the interpreters' performance, which often diverge from or even contradict the most common ethical principles, such as accuracy or neutrality. This study consists of a thorough 
analysis of the protocols used in the company Dualia Teletraducciones and a critical reflection from an ethical perspective on the adjustments suggested in them regarding the traditional role of the interpreter.

\section{Etični vidiki protokolov za telefonsko tolmačenje}

Ključne besede: telefonsko tolmačenje, protokoli, etika, veščine, vloga tolmača

Telefonsko tolmačenje postaja čedalje pomembnejša oblika tolmačenja $\mathrm{v}$ javnih službah v Španiji zaradi številnih prednosti, ki jih ponuja. Fernández Pérez (2012) je uvedel klasifikacijo posebnih veščin, ki jih morajo usvojiti telefonski tolmači glede na njihovi poglavitni nalogi, ki ju imajo pri tovrstnem tolmačenju: koordinirajo pogovor in prevajajo. Poleg teh veščin morajo telefonski tolmači nujno poglobljeno razumeti in natančno slediti protokolom, ki so zasnovani in razviti za različne stranke telefonskega tolmačenja, kot so protokoli, ki se uporabljajo za klice v sili.

Pričujoči prispevek se podrobneje posveča preučevanju prav teh protokolov. Naše izhodišče so specifikacije, ki jih je treba izdelati za delo tolmačev, ki se neredko razlikujejo ali so celo $\mathrm{v}$ nasprotju $\mathrm{z}$ najpogostejšimi etičnimi načeli, kot sta zvestost ali nevtralnost. Raziskava obsega natančno analizo protokolov, ki jih uporablja podjetje Dualia Teletraducciones, in kritični premislek (z etičnega vidika) o predlaganih prilagoditvah protokolov v zvezi s tradicionalno vlogo tolmača.

\section{Raquel Lázaro-Gutiérrez}

Raquel Lázaro-Gutiérrez es Profesora Contratada Doctora en el Departamento de Filología Moderna de la Universidad de Alcalá e imparte docencia en el Grado en Lenguas Modernas y Traducción y el Máster Universitario en Comunicación Intercultural, Interpretación y Traducción en los Servicios Públicos. Es miembro del Grupo de Investigación FITISPos-UAH desde el año 2001 y Vicepresidenta de la Asociación Europea ENPSIT. Ha sido IP en varios proyectos como "Análisis de ataques contra la imagen en interpretación telefónica" (2020-2021), "Diseño, compilación y análisis de un corpus multilingüe de interacciones mediadas sobre asistencia en carretera" (2018) e "Investigación sobre necesidades comunicativas y traducción e interpretación en centros penitenciarios" (2015). 


$\begin{array}{ll}\text { Dirección: } & \text { Dpto. Filología Moderna, } \\ & \text { Universidad de Alcalá, } \\ & \text { Colegio San José de Caracciolos, } \\ & \text { Calle Trinidad, } 3 \\ & 28801, \text { Alcalá de Henarees (Madrid), España } \\ & \text { raquel.lazaro@uah.es }\end{array}$

\section{Emilia Iglesias-Fernández}

Emilia Iglesias-Fernández es profesora titular de Interpretación en la Facultad de Traducción e Interpretación de la UGR. Ha participado en proyectos de investigación nacionales y europeos en los que explorado las características paralingüísticas de los intérpretes y cómo pueden favorecer evaluaciones de calidad. Sus estudios multidisciplinares de interpretación y fonética también se han enfocado en la transmisión de empatía en entornos médicos. Ha aplicado sus conocimientos a la audio descripción de largometrajes para discapacitados visuales. También ha revelado cómo la prosodia incide en la gestión de la interacción en interpretación telefónica.

Dirección:

Facultad de Traducción e Interpretación

Universidad de Granada

Calle Puentezuelas, 55

18071, Granada, España

Correo electrónico: emigle@ugr.es

\section{Gabriel Cabrera-Méndez}

Licenciado en Traducción e Interpretación por la Universidad de Granada. Traductor jurado e intérprete simultáneo de conferencias. Compagina su actividad profesional como traductor e intérprete con la docencia en la Universidad de Alcalá. Ha ostentado la vicepresidencia de ANETI (Asociación Nacional de Empresas de Traducción e Interpretación), es miembro del Comité 174 de redacción de normas ISO sobre traducción e interpretación, responsable de la calidad de la interpretación telefónica en Dualia Teletraducciones. Miembro del grupo de investigación FITISPos y autor de Mamá, quiero ser intérprete, entre otras publicaciones.

Dirección:

$\begin{array}{ll} & \text { Universidad de Alcalá, } \\ & \text { Colegio San José de Caracciolos, } \\ & \text { Calle Trinidad, 3 } \\ & \text { 28801, Alcalá de Henarees (Madrid), España } \\ \text { Correo electrónico: } & \text { gabriel.cabrera@uah.es }\end{array}$

